\title{
Esophageal Adenocarcinoma by AJCC v8 Clinical Stage
}

National Cancer Institute

\section{Source}

National Cancer Institute. Esophageal Adenocarcinoma by A/CC v8 Clinical Stage. NCI

Thesaurus. Code C133401.

A term that refers to the clinical staging of esophageal adenocarcinoma according to the American Joint Committee on Cancer, 8th edition. 